\title{
LAS CUÑAS MOTRICES EN EDUCACIÓN INFANTIL PERMITEN UN CUERPO PRESENTE EN EL AULA
}

The cuñas motrices in children education allow a body present in the classroom A cuñas motrices na primeira infância educação autorização um corpo presente em sala de aula

\section{Nuria Santamaría Balbás}

Universidad de Valladolid. Facultad de Educación de Palencia. Telf.: +34-616670616. Correo Electrónico: nsantamaria@educa.jcyl.es

\section{Resumen}

A través del análisis de los horarios en educación infantil, el catedrático Marcelino Vaca Escribano detecta que hay "diferentes presencias corporales en la escuela", y para favorecer un equilibrio entre dichas presencias, propone el uso de unas prácticas corporales que denomina "Cuñas Motrices". Con este objetivo, como tutora y especialista en educación física en una escuela pública, comienzo a desarrollar en el aula un proyecto de investigación, que se ha extendido a lo largo de nueve cursos escolares, en los que las cuñas motrices han logrado otros muchos objetivos, como la mejora del clima del aula y de la atención, la estimulación de las inteligencias múltiples o la compensación de la quietud, entre otros.

Para lograrlo, empleo herramientas como yoga, meditación, relajación, respiración consciente, masajes y el poder del tacto, trabajo con la energía, que se aplican en diferentes momentos de la jornada escolar, partiendo siempre de la escucha activa del maestro, para atender las curvas neurofisiológicas, los niveles de estrés y los ciclos de atención, pero también los deseos y las pulsiones de nuestros alumnos; dando así presencia a todos los cuerpos (físico, mental, emocional, espiritual).

Palabras clave: cuerpo, escuela holística, escucha activa, educación infantil.

\begin{abstract}
Through the analysis of hours in early childhood education, Professor Marcelino Vaca Escribano detects that there are "different body presences in school", and to promote a balance between these presences, he proposes the use of corporal practices that he calls "Cuñas Motrices". With this objective, as tutor and specialist in physical education in a
\end{abstract}


public school, I started to develop a research project in the classroom, which has been extended over nine school years, in which the driving wedges have achieved many other objectives, such as improving classroom climate and care, the stimulation of the multiple intelligences, the compensation of stillness, etc.

To achieve this, tools such as yoga, meditation, relaxation, conscious breathing, massage and the power of touch, energy work are used; they are applied in several times through the school day, always taking the teacher's active listening as the starting point, to pay attention to neurophysiological curves, stress levels and attention cycles, but also to our pupils' desires and urges; thus we can give presence to all the bodies (physical, mental, emotional and spiritual).

Keywords: body, holistic school, active listening, child education.

\section{Sumário}

Através da análise dos horários na educação infantil, o professor Marcelino Vaca Escribano detecta que há "diferentes presenças corporais na escola" e promover um equilíbrio entre essas presenças, propõe o uso de uma práticas corporais chamados "calços Motrices". Com este objectivo, como tutor e especialista em educação física em uma escola pública, comecei a desenvolver um projecto de investigação na sala de aula, que se espalhou por todo nove cursos escolares, em que as cunhas de condução alcançaram muitas outras metas, como a melhoria do clima de sala de aula e cuidado, estimulação das inteligências múltiplas, a compensação de quietude, etc. Para conseguir isso, use ferramentas como ioga, meditação, relaxamento, respiração consciente, massagem e o poder do toque, trabalho de energia, que se aplicam em diferentes momentos do dia escolar, sempre com base na professor escuta ativa para atender a curvas neurofisiológicos, os níveis de estresse e ciclos de atenção, mas também os desejos e impulsos de nossos alunos; dando assim a presença a todos os organismos (física, mental, emocional, espiritual).

Palavras Chave: o corpo na escola, escola holística, escuta ativa, Educação infantil. 


\section{1.- Introducción. Precursores del estudio}

A lo largo de esta introducción, voy a tratar de dar respuesta a preguntas que considero esenciales, para poder comprender el desarrollo de este artículo:

- ¿Cómo conozco las “Cuñas Motrices”?

- ¿Cómo va teniendo un interés progresivo en mi trabajo como maestra?

— ¿Cómo surge hacer de las cuñas motrices un objeto de estudio?

\section{1.- Mi primer encuentro con la expresión "cuña motriz"}

Todo comenzó en la Facultad de Educación de Palencia, donde me formé en la especialidad de Educación Física, en la asignatura que Marcelino Vaca impartía llamada: "Educación Física en Educación Primaria" (E.F. en E.P.), estábamos en el año 1994. Trabajando el concepto de lección, dentro de la elaboración de una Unidad Didáctica Marcelino expone: "Quisiera insistir que estas clases de una hora no son el único modo de desarrollar el área sino que, la coordinación del profesorado, tanto de ciclo como de etapa, pueden proporcionar otras situaciones educativas desde las que intervenir", (Vaca M.J. Apuntes de la asignatura "E. F. en E. P.") haciendo referencia a las Cuñas Motrices.

\section{2.- ¿Qué son las Cuñas Motrices y cómo surgen?}

Las Cuñas Motrices nacen, del gran interés que ya por el año 1977, Marcelino Vaca como maestro en el País Vasco, se planteaba en torno a la oportunidad de la motricidad en las aulas y su contribución a la interdisciplinariedad de estos aprendizajes. Para poder poner de manifiesto, que lo corporal expresa en buena medida lo que le ocurre al niño y a la escuela; comienza un estudio en el que observando, filmando y analizando sobre horarios reales, identifica diferentes presencias corporales, que se van sucediendo a lo largo de la jornada escolar (Vaca et al. 2013:12-15):

- Cuerpo silenciado: algunas de las tareas exigen que el cuerpo y su motilidad pasen desapercibidos, que se silencien.

- Cuerpo suelto: en los recreos en los se permite que el cuerpo esté suelto, a su aire.

- Cuerpo implicado, global o parcialmente: cuando las tareas escolares permiten la presencia del movimiento coordinado, incluso de la habilidad y destreza motriz... 
- Cuerpo instrumentado: a veces la tarea se presta a la utilización del cuerpo, de forma global o segmentaria, para ejemplificar corporalmente conceptos utilizados...

- Cuerpo expuesto: situaciones educativas que nos ponen ante la mirada de los otros...

- Cuerpo objeto de atención: por otra parte, la jornada escolar va desvelando necesidades corporales de descanso, de sueño, de alimentación, de higiene...

- Cuerpo objeto del tratamiento educativo: lo corporal, el cuerpo y la motricidad, se va instalando también como un área de expresión y de experiencia.

Con todas estas identificaciones, Marcelino Vaca se plantea la necesidad de atender la presencia del cuerpo en la escuela y, entre otras cuestiones, habla de una situación educativa particular "la Cuña Motriz": "Son momentos generalmente cortos (cinco o diez minutos), pero que realizados todos los días, suponen una interesante colaboración con los aprendizajes que pretendemos que logren los alumnos... y que vienen a ser una especie de bisagra entre dos momentos de exigencia, en los que se ha solicitado que "el cuerpo pase desapercibido" (Vaca, M.J. 2002:54). Con estas herramientas se pretende lograr un doble objetivo: dar una respuesta educativa al ámbito corporal y la generación del ambiente que reclaman los procesos de enseñanzaaprendizaje y su evaluación. Esta idea, ha ido evolucionando con el tiempo, como veremos en el apartado 2.

Desde el primer momento en que oí hablar de las cuñas motrices, este concepto me sedujo poderosamente, pues nos abría otras puertas a los especialistas de educación física, ya que no solo podríamos hacer un tratamiento educativo del cuerpo y la motricidad desde nuestra área, sino que además lograríamos intervenir en otros momentos y en otras materias.

1.3.- Un proyecto emblemático en un centro escolar: "Cuñas Motrices para mejorar la convivencia y el éxito escolar" (cursos 2009-2012)

En el año 2009, en el Centro de Educación Infantil y Primaria (C.E.I.P.) "Domingo Viejo" de Melgar de Fernamental -Burgos- (España); en el que trabajaba como maestra y especialista de E.F., fue en el que pude hacer realidad esta práctica pedagógica por primera vez, gracias a un proyecto colaborativo de centro, desarrollado para dar respuesta al cambio de jornada escolar. Denominamos al proyecto: “Cuñas Motrices para mejorar la convivencia y el éxito escolar" dando cabida a la doble función de las 
cuñas motrices: lograr la disponibilidad e implicación que los aprendizajes les reclaman a los alumnos y desarrollar procesos de enseñanza-aprendizaje específicos sobre el ámbito corporal.

\section{4.- La necesidad de indagar en el interés educativo de las cuñas motrices convierten el proyecto en una investigación}

Esta necesidad de indagar en las Cuñas Motrices como objeto de investigación y por supuesto de acción surge, por un lado, de la satisfacción que supuso trabajar en equipo con todo un claustro de profesores inquietos por una misma preocupación, preocupación que aborda el cuerpo y su motilidad en el aula y, con el objetivo de descubrir los efectos de la fatiga escolar y la pertinencia de atender las curvas fisiológicas.

Y por otro lado, son mis propias necesidades docentes dentro del aula las que me llevan a embarcarme en este continuo e inquieto deseo de saber, puesto que desde hace años compagino la función de tutora y especialista de educación física.

Estos fueron los acicates que me ayudaron a ampliar la visión del potencial educativo que nos ofrecían las cuñas motrices, como forma de entender, escuchar y de estar con nuestros alumnos. Las cuñas motrices se revelaban como un campo de acción y estudio... Y con este afán, me sumergí de lleno en la coordinación del "Seminario de formación", ofreciendo un nuevo enfoque, a través del trabajo de un grupo de tres cuñas motrices que se presentaban mensualmente y que abordaban actividades de: "movilidad articular y elongación muscular; control corporal y postural y ejercicios de respiración y relajación"

Las cuñas motrices, fueron encontrando un lugar cada vez más relevante en el aula, pues expresan una gran implicación por parte del alumnado, disposición, sosiego, creatividad, interacción, extrapolación de las experiencias, dando lugar a innumerables anécdotas y reflexiones personales. Como resultado de todo este trabajo y de la formación recibida, a partir del curso 2010-2011, el proyecto se hizo extensivo a todo el centro escolar, incluyendo las cuñas motrices en el horario oficial del centro y en el Proyecto Educativo (P.E.), logrando así un tercer objetivo: "Qué las cuñas fueran un recurso para el intercambio profesional y el trabajo compartido".

Este deseo de seguir indagando fue creciendo y así, a lo largo del curso 2011-2012, decidí dedicarme en cuerpo y alma a la realización del TRIT (Trabajo de Investigación Tutelado), investigación que creó los cimientos de una Tesis Doctoral: 
"Incidencia de las cuñas motrices y psicomotrices en la construcción de otra escuela", desarrollada entre los cursos 2012-2015, en un nuevo destino: el C.E.I.P. "María Teresa León" de Ibeas de Juarros (Burgos).

\section{2.- Recorrido de las cuñas motrices entre los cursos 2009-2018}

\section{1.- El proyecto se transforma gracias a la constante I-A}

La idea de partida, es que las cuñas motrices, son una serie de técnicas trabajadas de forma consciente, corpórea y vivencialmente, que realizaremos para facilitar al alumnado la transición de unos momentos a otros en la jornada escolar, favoreciendo con ello mejores niveles de atención y escucha. Sin embargo, con el análisis y la puesta en práctica de las cuñas a lo largo de estos nueve cursos escolares, me voy percatando, de que todas estas experiencias amplifican inimaginablemente mis expectativas, pues también nos van a otorgar espacios de sosiego, de concienciación y compensación corporal, de autoconocimiento, de contacto con el otro, de emociones expresadas, de aprendizajes transferidos, de duelos sanados, de nuevas relaciones forjadas. En esta transformación inciden tanto mi proceso formativo y personal, como todas las vivencias que voy teniendo en la escuela, a lo largo de estos nueve años en los que he desarrollado el proyecto; las cuales van impulsando y determinando una importante transición; sintiendo cómo el concepto de Cuña Motriz se amplia, abarcando todo un nuevo amplio espectro de disciplinas, que me permiten atender las necesidades que voy detectando en los niños.

De esta manera, estos paréntesis tan balsámicos, que le ayudan al alumno a descongestionarse, se van enriqueciendo con nuevas perspectivas a lo largo de todos estos años de exploración personal y de aplicación en el aula, que nos van conduciendo a nuevos territorios, pues van surgiendo cuñas en las que no hay motricidad, ni desplazamiento, ni intercambio tónico. $\mathrm{Y}$ así se va transformando el proyecto, alternándose el trabajo de cuñas corporales y motrices, con otras que se centran en el sentimiento cenestésico, un ámbito corporal conectado al mundo de las sensaciones y los sentimientos, que envuelve al niño, a su cuerpo, su motricidad, sus emociones, su identidad, su espiritualidad, sus búsquedas, sus relaciones. 


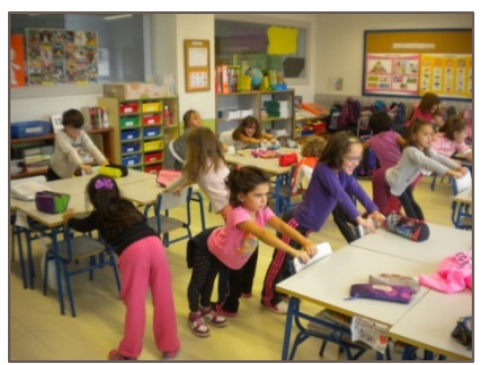

Foto 1

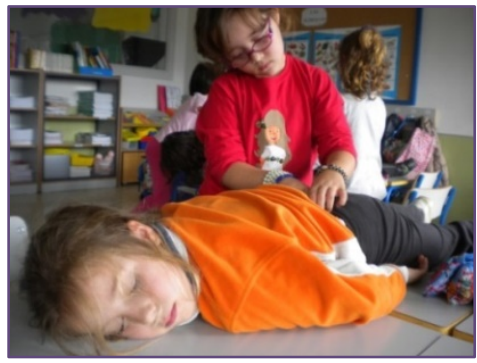

Foto 2

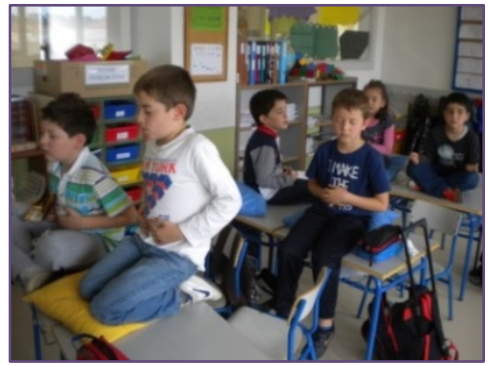

Foto 3

Por lo tanto, las cuñas realizadas atienden a todo el ámbito corporal. Y así la noción de Cuña Motriz crece y prospera en un concepto mucho más amplio; ya que pretendemos ejercer un saludable dominio sobre toda nuestra entidad biopsicosocial, teniendo en cuenta el tratamiento psicológico, biológico, fisiológico y neurológico del niño.

Esta transformación se va produciendo para poder acoger las diferentes realidades que advierto en los niños, niños que ya en educación infantil se enfrentan a gran cantidad de estímulos perturbadores, a influencias nocivas por altos niveles de actividad o exigencia, o por diversas y a veces trágicas situaciones familiares. Todo ello les provoca estar sometidos a umbrales muy altos de ansiedad y tensión, tanto física, mental como emocional. Con las cuñas motrices, se pretende no solo liberar al cuerpo de estas tensiones, sino también sosegar la mente y las emociones, sintiendo que cada vez es más necesario buscar espacios interiores de silencio en las aulas.

En las cuñas utilizamos el cuerpo, como instrumento para implicar a la mente y, conseguir así un buen desarrollo y armonía, pues entendemos que a través de la toma de conciencia de la corporeidad, podemos ir modificando la mente, refrenando los pensamientos y conquistando estados de conciencia y sosiego más profundos.

Con las cuñas pretendemos estar atentos a un cuerpo vivo, a su expresividad. Es decir, a su capacidad de decir quién somos, qué nos preocupa, emociona, confunde o daña. Todo ello mediante actividades físicas de atención consciente, de respiración, de relajación, de meditación, de canalización energética, de contacto íntimo consigo mismo y con el otro, pudiendo así descodificar la repercusión que sobre el cuerpo tienen las reacciones mentales o emocionales insanas, evitando bloqueos, tensiones y sus consiguientes implicaciones en los aprendizajes. 


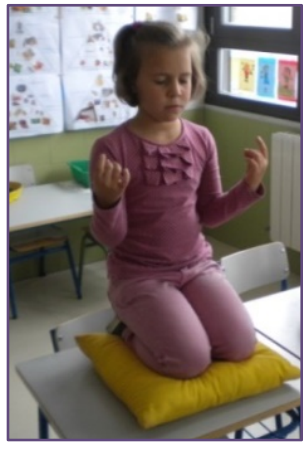

Foto 4

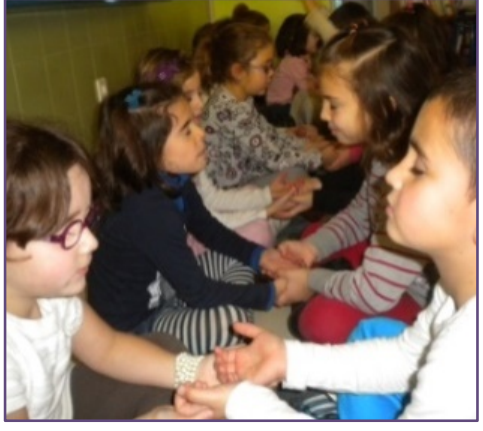

Foto 5

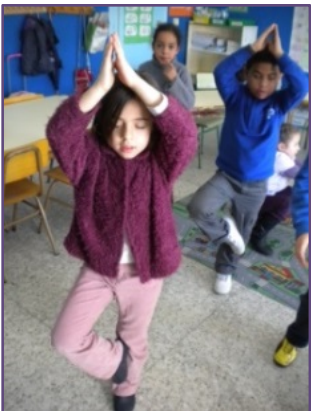

Foto 6

Luego es un planteamiento en el que el trabajo con el cuerpo, la mente y las emociones, fluyen paralelamente; se tornan herramientas necesarias, íntimas e inseparables para lograr la evolución consciente que buscamos; para reintegrar y equilibrar todos los planos de la persona, acrecentando así su conciencia e interiorización. Personalmente me interesa abordar y proyectar este trabajo de desarrollo holístico del niño en la escuela, pues me siento en comunión interna entre mis propias necesidades y experiencias de evolución vividas y las detectadas en mis propios alumnos; sintiendo así la responsabilidad de compartir todas estas herramientas que llegan a mi vida, con ellos; para poderles aportar escenarios, estrategias o instrumentos con los que puedan escucharse, valorar y reconocer sus talentos, superar sus duelos, conocerse interiormente, sanarse física y energéticamente, tocar y dejarse tocar, sentir y dejarse sentir, aprender a pensar, a mirar, a sentir, a buscar respuestas diversas, a descubrir el valor de la belleza, de las cosas hechas con pasión y con amor. Es como partir de sus conocimientos previos más íntimos, un saber previo que bulle y busca compañía, comprensión, orientación, para entonces construir la casa.

Me gustaría insistir en la idea de que cuando ofrezco unas herramientas o facilito un ambiente, en el que se den situaciones que al niño le permitan descubrirse, escucharse, sentirse, sanarse, etc. mi papel es el de acompañar en el proceso. Y este planteamiento educativo, conlleva un indispensable requisito: la formación personal del docente.

\section{2.- Evolución en la aplicación de las cuñas motrices en el aula}

Uno de los aspectos que se modifica tajantemente a lo largo de estos nueve cursos, es $\boldsymbol{e l}$ momento en el que aplicar la cuña, ya que en el proyecto primigenio, las cuñas se venían trabajando en los cambios de asignatura, a modo de bisagra entre clase y clase. 
Sin embargo en la actualidad, las cuñas ya no se aplican únicamente en los intercambios de materias, sino que gracias a la indagación y al respeto inferido en su aplicación, estas me han ido demostrando que tienen la gran cualidad de adaptarse a las necesidades de cada aula, e incluso de cada alumno, siendo el propio niño y la clase en su conjunto, quien con su lenguaje corporal nos va diciendo las necesidades que tiene en cada momento. Desde este punto de vista, desde esta forma de plantear el trabajo con las

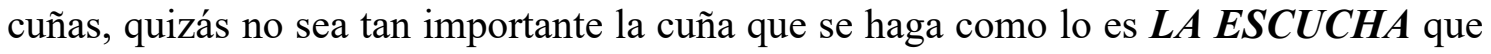
se realice.

La I-A realizada a diario en el aula, me ha hecho ver que hay grupos que a primera hora de la mañana necesitan tonificación o activación y otros que, sin embargo, precisan una relajación; grupos que llegan del recreo con una gran agitación y reclaman una vuelta a la calma en este momento; clases con una gran exigencia que demandan un corte para relajar tal intensidad; o incluso grupos en los que unos alumnos necesitan una activación y otros una relajación y las cuñas motrices lo permiten.

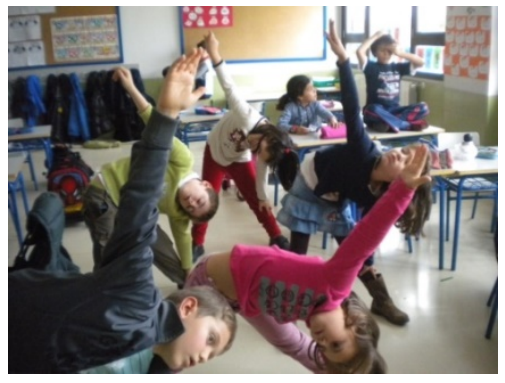

Foto 7

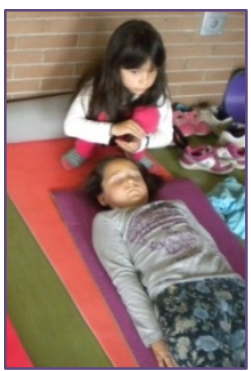

Foto 8

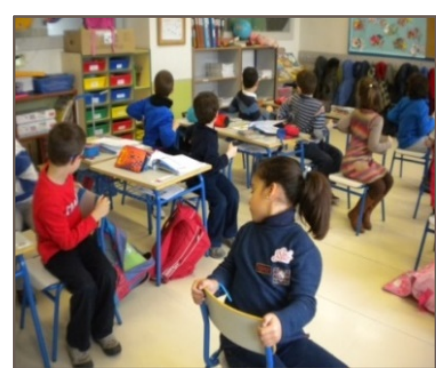

Foto 9

Sin embargo, dentro de esta flexibilidad desarrollada día a día en la aplicación de las cuñas, van surgiendo otras que se establecen en un momento concreto y, gracias a las experiencias vividas y a los beneficios detectados, se mantienen a día de hoy. Me refiero a "La cuña de bienvenida" y la realizada "Antes de una evaluación".

"La cuña de bienvenida" surge para mostrarles una forma más amable de dar la bienvenida al día, sin prisas, sin tensión, sin reproches. De esta forma, comienzo cada día de la semana con una cuña motriz diferente: los lunes- termómetro emocional; los martes- respiración consciente; los miércoles- agradecimientos; los jueves- meditación guiada y los viernes - meditación a través del canto.

"La cuña de antes de la evaluación" pretende eliminar o reducir la tensión y la sensación de fracaso, con la que muchos alumnos se enfrentan a los exámenes, 
propiciando una "purificación física, mental y emocional"; combinando un trabajo con la respiración consciente, la movilización articular y el Tapping, junto con frases positivas.

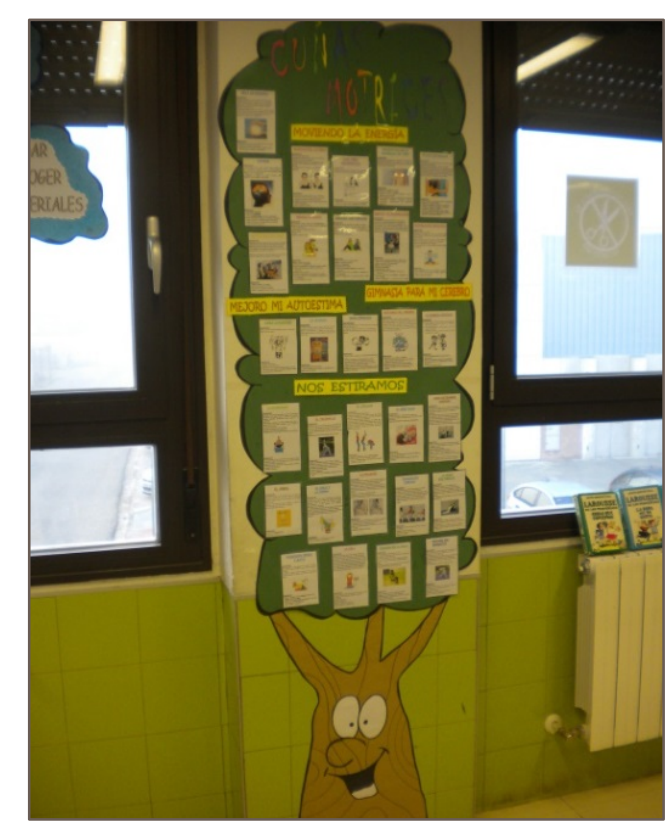

Foto 10: “Árbol de las Cuñas” (curso 2014-2015)

En el curso 2014-2015, la forma de trabajar y de presentar las cuñas se modifica contundentemente, surgiendo en septiembre de 2014, el “Árbol de las Cuñas", donde están impresas parte de las cuñas que he ido elaborando y seleccionando a lo largo de estos siete años. Algunas de estas cuñas están presentadas de forma genérica, pues luego son ampliadas en diferentes momentos $\mathrm{y}$ propuestas en el aula.

Este cambio de metodología, responde al deseo de trabajar las cuñas desde la "autogestión", profundizando así en esta perspectiva "corporal" y "somática"; para respetar y aceptar el propio dialecto corporal del niño, es por este motivo, por el que muchas cuñas no pueden tener horario fijo, pues son fruto de una lectura de la expresividad corporal, ya que el cuerpo dice en cualquier momento, solo es cuestión de saber y poder escucharlo...

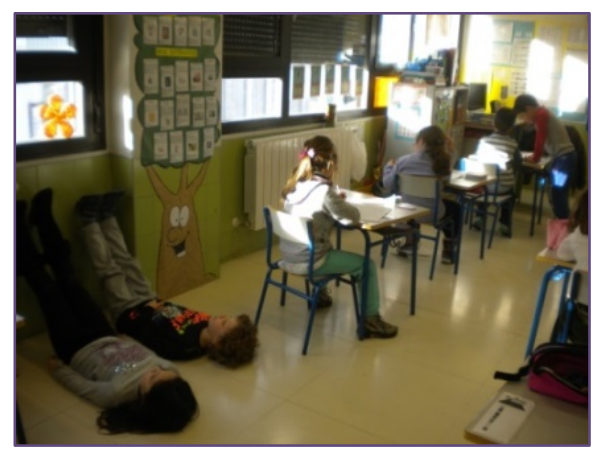

Foto 11

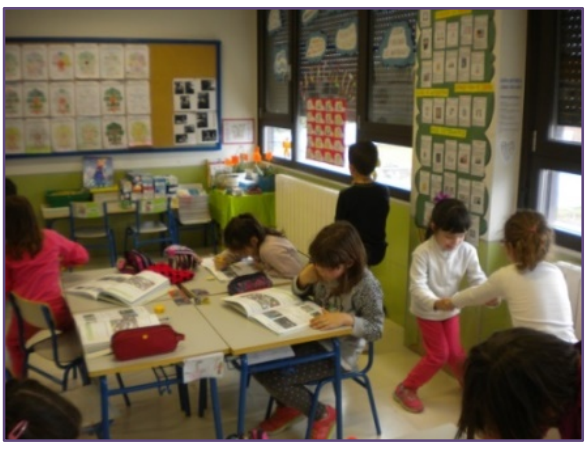

Foto 12

Con este planteamiento pedagógico, se pretende llamar la atención sobre la preponderancia que tiene el cuerpo como elemento de generación de conocimiento. 


\section{3.- Objetivos del estudio y exposición de los datos}

\section{3.a. Objetivos del estudio}

La presente investigación, tiene una primera parte centrada en un proyecto de I-A colaborativa, realizado a lo largo de tres cursos escolares en el C.E.I.P. "Domingo Viejo" de Melgar de Fernamental, (2009-2012), y una segunda parte, centrada en un nuevo proyecto de I-A, llevado a cabo en el C.E.I.P. "María Teresa León” de Ibeas de Juarros, en Burgos, durante los cursos 2012-2015, aunque el proyecto continua a día de hoy. Entre ambas partes ha habido una continuidad pues como explica Inma Cases: "Toda investigación se gesta mucho tiempo antes de su concreción y va acumulando la experiencia práctica, las creencias, los supuestos escondidos y tantas otras vivencias individuales y colectivas" (Imbernón, 2002: 119).

El objeto de investigación, parte del diagnóstico de una situación real surgida en nuestras aulas en el que buscamos alternativas para dar respuesta a los nuevos requisitos que suponía un cambio de horario en el centro. Para lograrlo buscamos estrategias didácticas y pedagógicas, que nos ayudaran a atender las necesidades fisiológicas, corporales, mentales y emocionales de nuestros alumnos, mediante diversas herramientas y técnicas corporales.

Para ayudarnos en el análisis y desarrollo del proyecto, se realizó una revisión documental tanto del currículo oficial como en la literatura educativa que nos llevó a concretar los objetivos de la tesis:

- OBJETIVO 1: Comprobar la aplicabilidad del proyecto de I-A colaborativa realizado en el C.E.I.P. "Domingo Viejo", en otros contextos.

- OBJETIVO 2: Seguir indagando en el interés educativo de las Cuñas Motrices, lo que se concreta en comprobar si las Cuñas Motrices:

- Colaboran activamente en la mejora del "ambiente de clase".

- Compensan la quietud, el cansancio, el nerviosismo, la tensión o la falta de atención.

- Estimulan diferentes inteligencias; construyen aprendizajes; desarrollan capacidades y hacen crecer las competencias clave.

- Construyen aprendizajes que mejoran la vida del aula y tienen uso fuera de ella. 
- Contribuyen a enriquecer el currículo y favorecen la inclusión de metodologías activas.

- Ayudan a enriquecer las relaciones familia-escuela.

- Reclaman un nuevo rol del maestro y un desarrollo profesional determinado.

\section{3.b.- Exposición y análisis de los datos del estudio}

En relación al primer objetivo que nos proponíamos: "Comprobar la aplicabilidad del proyecto de I-A colaborativa realizado en el C.E.I.P. "Domingo Viejo", en otros contextos"; podemos decir que trasladar un proyecto que tantas alegrías y buenos resultados había dado en el colegio en el que nació, a otro centro escolar, tiene sus vicisitudes. No ha sido un camino fácil, pero ha habido muchas cosas positivas en el recorrido. El hecho de partir de un fecundo trabajo colaborativo, de unas experiencias positivas previas, de unas indagaciones que dieron como fruto el establecimiento de unos campos de interés educativo de las cuñas motrices, hacían prever una rápida trasmisión al nuevo contexto. Sin embargo, no se respalda ni se da el apoyo necesario para desarrollarlo como proyecto de centro en el colegio de Ibeas. De esta forma, sintiendo que la acción colaborativa, no puede tener la misma intensidad y profundidad que la desarrollada en Melgar, genera el comienzo de nuevos trayectos. Y en él van surgiendo nuevos aliados que colaboran en la transformación del mismo, produciéndose cambios curso a curso. El proyecto no se podía trasladar, había que reconstruirlo.

Con el análisis y la puesta en práctica, dentro de mi aula, de las cuñas en el colegio de Ibeas, me voy percatando de que todas las experiencias incrementan inimaginablemente mis expectativas, amplificándose los campos de interés detectados en Melgar. De esta forma, mediante la constante indagación, los datos y experiencias recogidas, se van consiguiendo lentos, silenciosos pero progresivos cambios en el centro. Muchos de los logros e innovaciones introducidas quizás no resulten espectaculares. Es más, muchos de ellos están documentados e identificados en otras tentativas. Pero su importancia radica en que, ahora, proceden de las experiencias vividas y reelaboradas en el colegio de Ibeas de Juarros. Tratar de penetrar en la cultura escolar de un centro, para implementar un proyecto de este calado, que trabaja con una visión holística del niño, no es sencillo, se necesita infinita paciencia, ilusión, respeto, capacidad de revisión y decisión. 
Por otro lado, voy siendo más consciente de que el acto educativo es tan complejo, que nos impide dar como respuestas definitivas soluciones que hayan tenido un resultado positivo en un contexto anterior, pues los alumnos, los compañeros, la idiosincrasia del centro y las familias son diferentes. Sin embargo, podemos constatar a través de los instrumentos empleados en la recogida de datos: mediante las observaciones realizadas, los documentos elaborados, los estudios de casos y las anécdotas relatadas, cómo el proyecto cada vez está más asentado en el centro, cómo son los alumnos y sus familias las que reclaman su utilización, cómo es una seña de identidad en las aulas en las que se aplica, cómo Ibeas es un referente para otros centros de la provincia de Burgos, en la utilización de esta herramienta educativa.

Todo ello lo corroboran los datos hallados en las encuestas realizadas a los alumnos y a las familias y, que podemos ver tanto en las tablas resumen, en la mediana de los datos y el rango, en los resultados de aplicar el test U de Mann Whitney, (para ver si hay diferencias estadísticamente significativas entre colegios), y en el análisis elaborado para medir la satisfacción de los niños en ambos colegios. Tan solo hay diferencia en el caso del de clima de clase, en el que el p-valor es menor de 0,003, es decir que hay diferencia entre la puntuación obtenida en la suma de los ítems "si" en la encuesta en la categoría de clima de clase y el pertenecer a un colegio u otro. El resto de los p-valor son grandes, luego, aceptamos la hipótesis nula: por lo tanto la distribución de cada categoría es la misma entre colegios.

\begin{tabular}{|c|c|c|c|c|c|c|c|c|}
\hline & \multirow{2}{*}{\multicolumn{2}{|c|}{$\begin{array}{l}\text { General } \\
(n=112)\end{array}$}} & \multicolumn{4}{|c|}{ Colegio } & \multirow{3}{*}{$\begin{array}{c}p \text {-valor } \\
0,085\end{array}$} \\
\hline & & & & \multicolumn{2}{|c|}{ Ibeas $(n=46)$} & \multicolumn{2}{|c|}{ Melgar $(n=66)$} & \\
\hline P1_8 & Clima de la clase & 62 & $55 \%$ & 21 & $46 \%$ & 41 & $62 \%$ & \\
\hline P9_15 & Compensación corporal & 40 & $36 \%$ & 13 & $28 \%$ & 27 & $41 \%$ & 0,169 \\
\hline P16_23 & Inteligencia corporal cinestésica & 68 & $61 \%$ & 33 & $72 \%$ & 35 & $53 \%$ & 0,046 \\
\hline P24_28 & Éxito escolar & 73 & $65 \%$ & 29 & $63 \%$ & 44 & $67 \%$ & 0,692 \\
\hline P29_34 & Inteligencia intrapersonal e interpersonal & 75 & $67 \%$ & 30 & $65 \%$ & 45 & $68 \%$ & 0,743 \\
\hline P35_40 & Inteligencia intrapersonal (emociones y duelo) & 65 & $58 \%$ & 27 & $59 \%$ & 38 & $58 \%$ & 0,906 \\
\hline P41_45 & Inteligencia espiritual & 91 & $81 \%$ & 36 & $78 \%$ & 55 & $83 \%$ & 0,499 \\
\hline P46_50 & Hábitos alimenticios & 80 & $71 \%$ & 33 & $72 \%$ & 47 & $71 \%$ & 0,952 \\
\hline
\end{tabular}

* Los valores corresponden al SI

Tabla 1: Resultados generales y comparativos de los cuestionarios pasados a los alumnos

Teniendo en cuenta las limitaciones presentadas y todos los datos recogidos, queda reflejado que no existen diferencias significativas en la satisfacción de los 
alumnos, entre ambos centros escolares. Lo que demuestra que el nivel de satisfacción en ambos colegios es muy similar y excelentemente positivo, en torno al desarrollo de las cuñas motrices en el aula. Podemos entonces afirmar, que los datos confirman que el proyecto es extrapolable a otros contextos.

En torno al segundo objetivo propuesto: "Seguir indagando en el interés educativo de las Cuñas Motrices", lo que se concreta en comprobar si las Cuñas Motrices:

\section{A.- Colaboran activamente en la mejora del ambiente de clase}

A lo largo de estos años de puesta en práctica del proyecto en diferentes contextos, he comprobado que para favorecer un buen ambiente en el aula, se hace necesario la creación de un clima de respeto, de confianza, de serenidad; el cual modifica, de manera sustancial, las experiencias en el aula, el clima de intercambio y de aprendizaje y las relaciones internas y externas. Para lograrlo se han atendido los cuatro elementos clave en la interpretación del clima de un aula que nos propone Isabel Fernández García (Cita en Torrego et al. 2007:173-177) que son: el cuidado de los tiempos y los espacios; las interacciones, las programaciones didácticas y el rol del maestro.

En lo que se refiere a la organización de los tiempos, he transformado los horarios reales del aula para favorecer un acercamiento a las necesidades de los alumnos, escuchando y atendiendo sus estados de cansancio, tensión, falta de atención y humores emocionales. De forma que las cuñas nos permiten otorgar a los alumnos un tiempo para sí mismos, antes de adentrarles en materias que les exigen una fuerte descentración, que requiere de implicación y disponibilidad.

En la creación de este clima de aprendizaje, es necesario contar con espacios libres de amenazas, en los que el niño tenga la aceptación de sus compañeros y del maestro, lo cual hace imprescindible el cuidado de las interacciones. La puesta en práctica de las cuñas no solo no ha mejorado la relación y el conocimiento de uno mismo, sino que ha mejorado la relación con los demás, ya que al reducir la agitación, el estrés, la ansiedad o el nerviosismo, se ha colaborado en la corrección de reacciones desmesuradas y en consecuencia, en conflictos innecesarios. Todo ello nos ha ayudado en la generación de un clima de calma, respeto y sosiego, favoreciendo mejores relaciones en el aula, reduciéndose las discusiones y los conflictos; y además nos ha 
permitido que haya un tratamiento colaborativo en la construcción de las normas de convivencia.

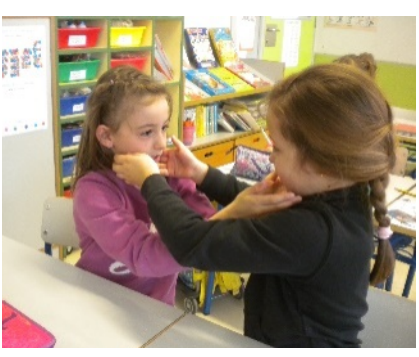

Foto13

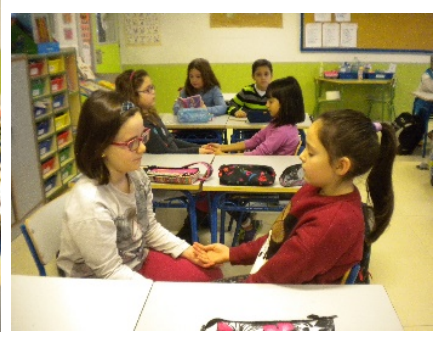

Foto14

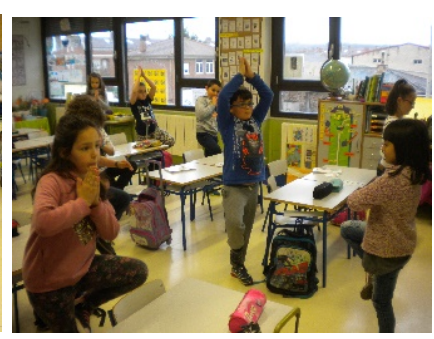

Foto15

\section{B- Compensan la quietud, el cansancio, la tensión o la falta de atención}

Este fue el primer motivo que nos llevó a aplicar las cuñas motrices en el aula. Para lograrlo se buscó la creación de ambientes de serenidad y respeto de los ritmos y pulsos personales, corporales y emocionales en la clase, mediante la puesta en práctica de cuñas que se vienen trabajando a modo de pequeñas intervenciones y en diferentes momentos de la jornada escolar, las cuales abonan el camino para favorecer un nivel óptimo de atención y reducción del nivel de tensión de nuestros alumnos.

La primera de las estrategias empleadas para lograrlo, ha sido elaborar horarios respetuosos que atiendan las curvas neurofisiológicas existentes en el aula. En esta atención estudiada de los tiempos, las cuñas motrices se van aplicando en diferentes momentos de la jornada escolar. Así las cuñas cada vez cobran más protagonismo en el aula y se convierten en tiempos de aprender, en situaciones educativas que se mezclan con otras materias escolares.

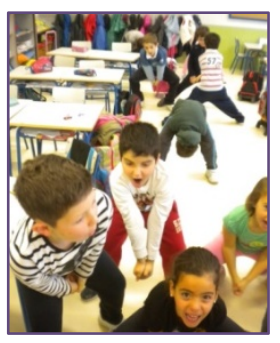

Foto 16

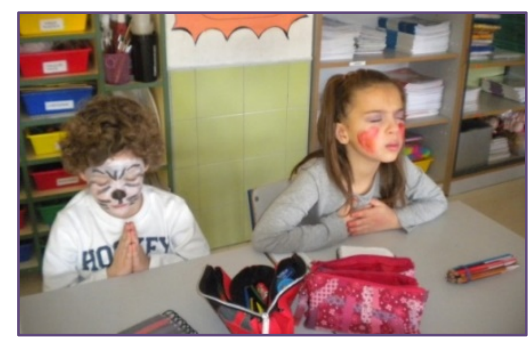

Foto 17

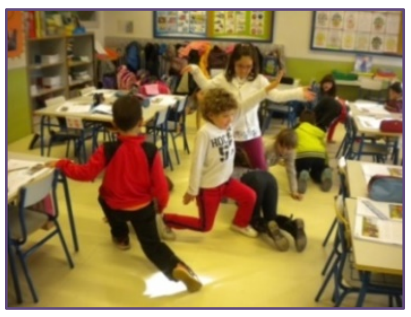

Foto 18

C.- Estimulan diferentes inteligencias; construyen aprendizajes; desarrollan capacidades y hacen crecer las competencias clave

A través del trabajo con las cuñas, se ha creado un entramado en el que se da cabida a todos los cuerpos: físico, mental, emocional y espiritual. Para lograrlo empleamos 
herramientas que nos ayudan a enfocar la atención, a estar en el presente, a respirar conscientemente, a tocar y ser tocados, a sanar nuestras dolencias, a movilizar las distintas cadenas musculares, a saber estar en un silencio interior; utilizando para lograrlo herramientas como: yoga, meditación: visualizaciones y canto de mantras, relajación, respiración consciente, masajes y el poder del tacto, trabajo con la energía y el contacto con la naturaleza (plantas, cristales, aromas). Bajo esta perspectiva, se hace imperante reivindicar la responsabilidad que tiene cada persona con respecto a su propia salud y al logro de su bienestar, recuperando legados ancestrales adormecidos y prácticas que le resultan naturales y familiares al cuerpo, a la mente y al espíritu.

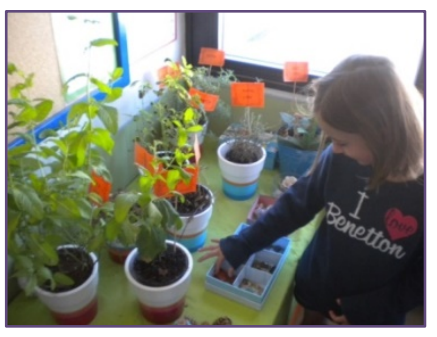

Foto 19

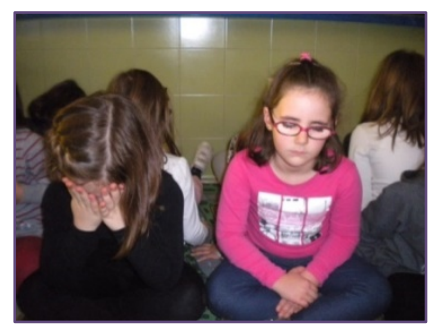

Foto 20

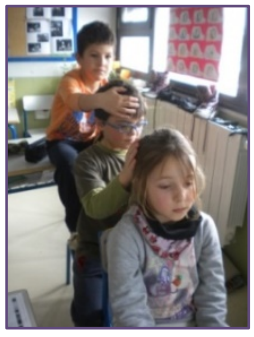

Foto 21

He ido constatando, a lo largo de toda la investigación como mediante la escucha activa realizada, las cuñas que tratan de compensar la quietud, las malas posturas y las tensiones generadas, son tan necesarias como las que propicien que se dé una quietud interior. De esta forma, vamos logrando que en el aula haya momentos y oportunidades para poder cerrar los ojos, para parar y escuchar, momentos de sosiego en los que descubrir que existe un gran espacio interior que puede ser habitado y desde el cual las cosas se pueden llegar a ver, vivir y sentir de una manera más apacible.

Gracias a estas prácticas corporales, se crea un espacio para la manifestación de los duelos personales, del llanto, para hablar de la muerte y de la vida, para compartir los dolores, pero también los deseos, los anhelos y los talentos. Y en el desarrollo de estas cuñas, que comienzan como algo breve, se despierta una necesidad en el niño y se generan vivencias con tanto interés y profundidad, que las convierten en lecciones.

\section{D.- Construyen aprendizajes que mejoran la vida del aula y tienen uso fuera}

A lo largo de estos nueve años hemos tratado de corroborar, curso tras curso, que las cuñas motrices aportan a los alumnos experiencias de aprendizaje que van más allá de la adquisición de conocimientos o de habilidades concretas, puesto que ofrecen también oportunidades reales de cambio para su formación y para su vida futura. Partiendo de 
esta premisa, tratamos de que los aprendizajes vayan más allá del aula, permitiendo la conexión de contextos formales e informales de aprendizaje.

Se hace necesario conocer el proyecto en profundidad, para comprender que no es solamente un método, que se trata de una praxis experiencial, en la que se conjugan objetivos propedéuticos, formativos y hasta terapéuticos. Y solo es posible con una ejercitación; que se extiende a todos nuestros cuerpos, para realizar una conquista de lo que somos más que lo que sabemos.

\section{E- Contribuyen a enriquecer el currículo y favorecen la inclusión de metodologías activas}

Este progresivo e incesante proceso de cambio y evolución pedagógica, acontecida gracias a todo el trabajo realizado a lo largo de estos cursos con las cuñas motrices, produce un crecimiento ramificado en todas las esferas de mi quehacer docente, provocando paralelamente, la transformación y perfeccionamiento de la programación didáctica de aula (programación globalizada de las áreas y trabajo alternativo al libro de texto), de las metodologías aplicadas (incorporando metodologías activas: rutinas de pensamiento, trabajo cooperativo) y del proceso de evaluación realizado.

Esta apertura pedagógica, me ha hecho abrir los ojos de una forma indescriptible, haciéndome ser muy crítica con mi propia práctica y con todo lo que me rodea; lo cual me provoca, en muchas ocasiones, una gran soledad pedagógica.

Todo este cambio ha exigido formación y una constante reflexión sobre la acción, la cual ha desplegado mi creatividad y me ha abierto a nuevas expectativas.

\section{F- Ayudan a enriquecer las relaciones familia-escuela}

Atender tanto las necesidades académicas como las fisiológicas, emocionales, corporales o sociales del niño, nos ha permitido la generación de aprendizajes más provechosos y la generación de ambientes más potentes que se filtran en el pensamiento del profesorado y en los ambientes familiares, pues todas las profundas y sanadoras experiencias vividas por el niño en la práctica de las cuñas, tienen repercusión en su contexto más próximo, su casa. Ello ha favorecido la apertura de nuevos espacios de encuentro con los padres, con los que se comparten intensas conversaciones, se intercambian preocupaciones e intereses mutuos, agradecimientos y consejos, peticiones y propuestas, compromisos y respeto. En la actualidad, he de reconocer que son mis 
principales aliados, junto a sus hijos. Son ellos quienes me siguen animando a desarrollar cada propuesta que les planteo, por "insólita" que sea. Todos los extraordinarios y terapéuticos momentos vividos con las familias, han duplicado el sentido de las cuñas motrices.

Cada solicitud de ayuda, cada muestra de confianza depositada, fortalece aún más mi relación y conexión con los niños y este encuentro me lleva a involucrarme más y más en su proceso vital.

Como se puede ver en los resultados generales de las encuestas realizadas a las familias, los padres están muy satisfechos con la utilización de las cuñas en el aula.

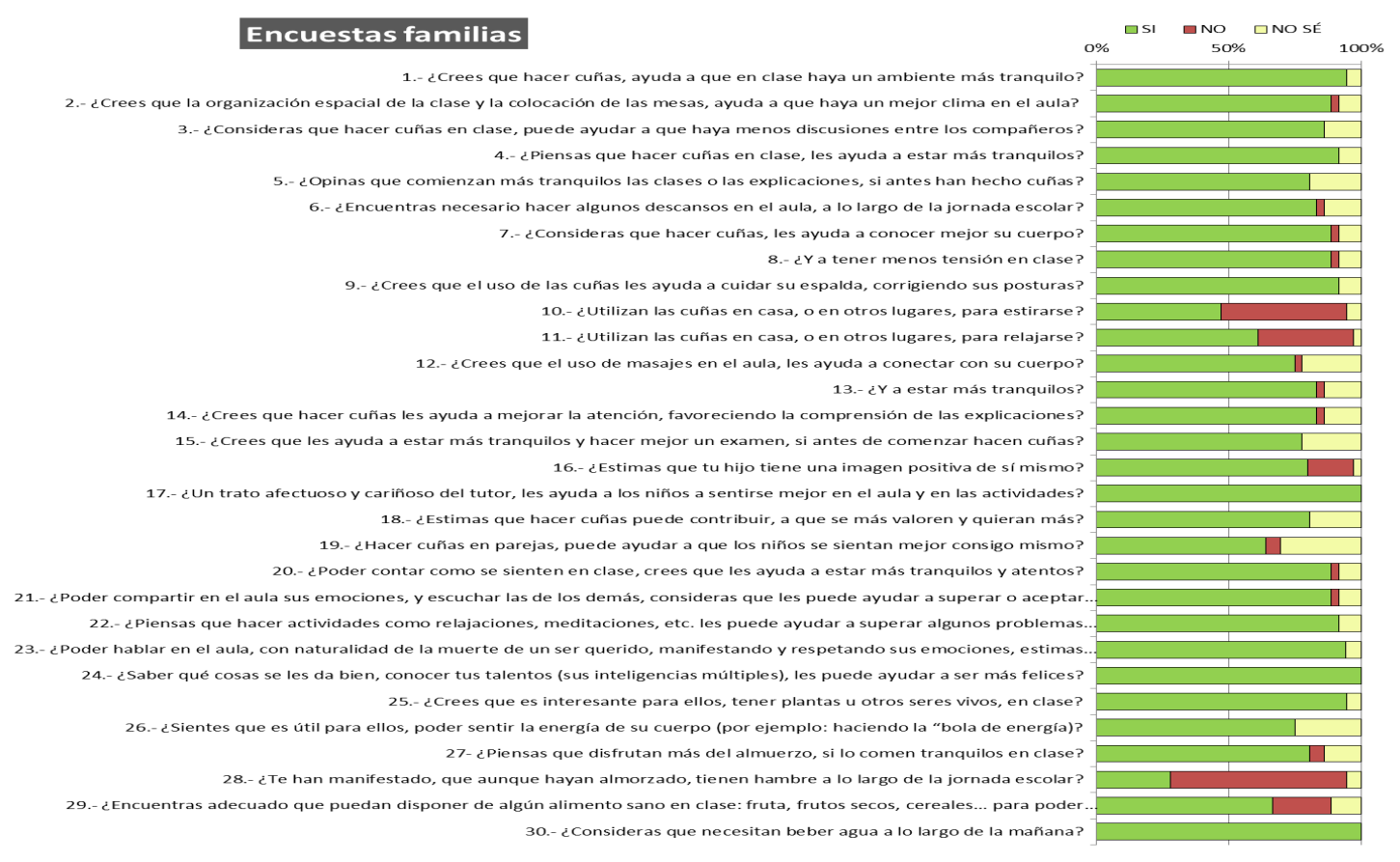

Tabla 2. Resultado de las encuestas realizadas a las familias del colegio de Ibeas de Juarros.

\section{G.- Reclaman un nuevo rol del maestro y un desarrollo profesional determinado}

La construcción de esta escuela particular, precisa de un docente no solo versado en programar, en destrezas metodológicas o en competencias disciplinares, sino también un docente capaz de estar disponible, en la escucha, en la mirada, en la acogida, siendo fundamental la conexión emocional que se haga con el grupo, que favorezca un clima de afecto y de respeto, en el que la ternura y el rigor no estén reñidos, sino en equilibrio. Para crear estos espacios de acción y de calma, se precisa por lo tanto, de un maestro 
que se desenmascare e involucre afectiva y físicamente. De esta forma, el aula pasa a ser un espacio de descubriendo interior y de desarrollo personal compartido.

El maestro se convierte en la principal herramienta pedagógica en la transmisión de las cuñas motrices. Tras la observación directa y las reflexiones de algunos compañeros a lo largo de estos años de investigación, se hacen evidentes las dificultades que muchos docentes tienen a la hora de exponerse corporal y motrizmente ante sus alumnos, surgen los miedos, las limitaciones, los bloqueos; lo que supone que también "el cuerpo" del maestro debe reeducarse y adaptarse, para poder así ser capaces de escuchar el "cuerpo" del niño, y comprender sus mensajes, demandas, necesidades o deseos.

En las encuestas realizadas a los maestros, podemos comprobar como la compensación de los niveles de cansancio y de atención, la mejora de las relaciones y del clima de clase, son los aspectos en que las cuñas motrices más les han ayudado.

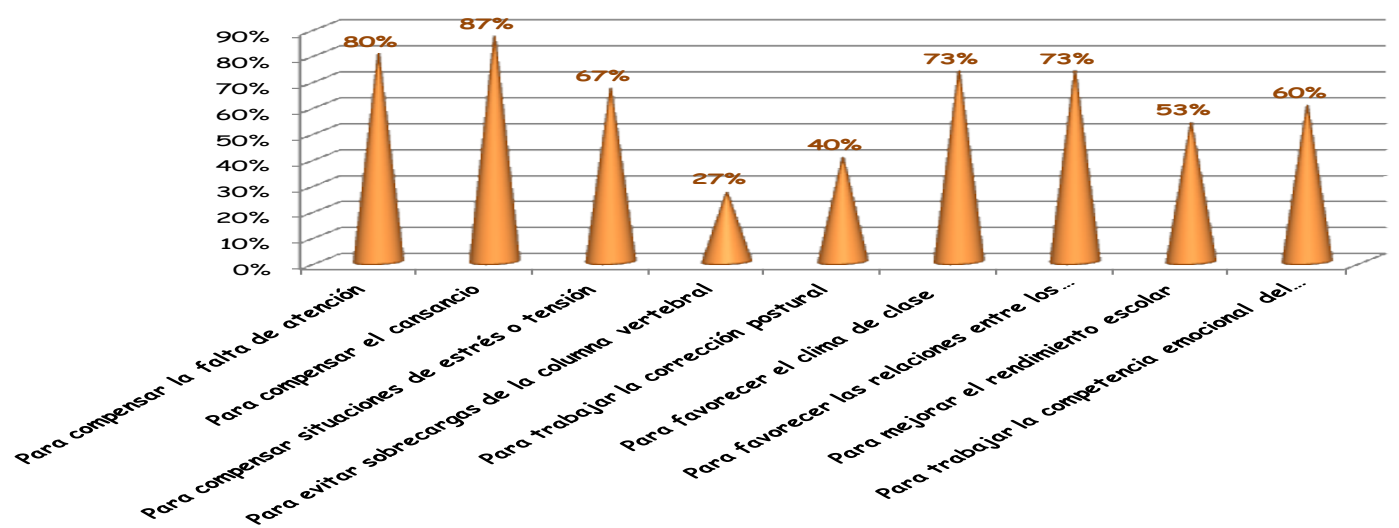

Tabla 3. Resultado de los aspectos en que han ayudado a los maestros las cuñas motrices

$\mathrm{Y}$ en cuanto a las dificultades encontradas por los maestros a la hora de aplicar las cuñas, los mayores obstáculos son: la falta de tiempo y de concentración del alumnado. 

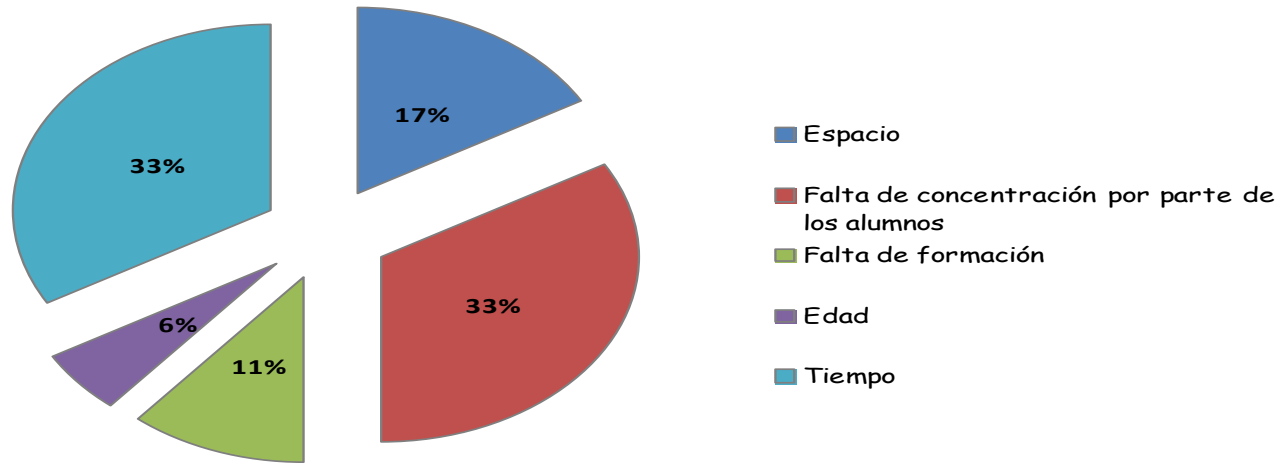

Tabla 4. Dificultades encontradas por los maestros en la aplicación de las cuñas motrices

\section{4.- Breves conclusiones}

Para ayudar a entender las dificultades encontradas y a encauzar las oportunidades que las cuñas motrices nos dispensan en la escuela, surge este esquema en torno a tres vértices: la educación corporal, la creación de ambientes y las nuevas relaciones establecidas; que a su vez provocan un cambio global en la metodología desarrollada y la consiguiente transformación de la figura del maestro:

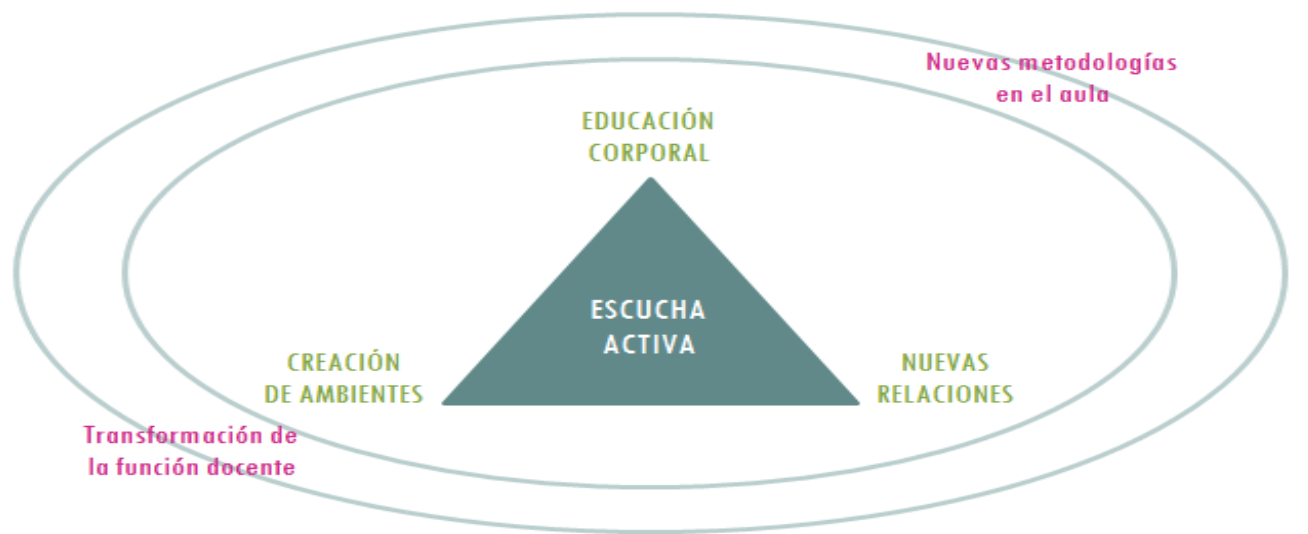

Tabla 5. Esquema para entender las dificultades encontradas y encauzar las oportunidades de las Cuñas Motrices

Este triángulo o las razones de los saberes que aportan las cuñas, se ha visto fortalecido, en el nuevo contexto de aplicación del proyecto, de la siguiente forma:

Educación corporal: a través de su "escucha activa" se conecta su tratamiento educativo con el resto de los aprendizajes, con las cuñas como hilo conductor; tratando de que el movimiento tenga un sentido, un propósito. 
Creación de ambientes: ofreciendo otras posibilidades para el desarrollo de las tareas propias de la escuela, a través de ambientes adecuados con los que conseguir la implicación y disponibilidad del niño para aprender.

Nuevas relaciones: creadas a partir del respeto, de la escucha observadora, de la atención amorosa y del acompañamiento.

Nuevas metodologías aplicadas en el aula: la escucha realizada hacia las necesidades e intereses de los niños, provoca un profundo cambio metodológico en el aula.

Transformación de la función docente: para poder desarrollar un proyecto en el que los cambios y la escucha son diarios, es necesario hacer reformas personales, que conllevan una innovación de la función del docente en la escuela.

A modo de breve resumen, diré que las Cuñas Motrices nos permiten elaborar un marco didáctico en lo que se refiere a la construcción de unos saberes motrices; pero las cuñas también nos posibilitan un marco pedagógico de creación de espacios de sosiego, escucha, respeto, calma y entendimiento, que favorecen la construcción de dichos saberes. Saberes que quieren ser un bien sin caducidad con recorrido del ciclo vital, para que tengan continuidad en el entorno más próximo al alumno, creando hábitos de prácticas saludables. Es por lo tanto, un proyecto en el PRESENTE para el FUTURO.

\section{Referencias bibliográficas}

Imbernón, F. y otros (2002). La investigación educativa como herramienta de formación del profesorado. Reflexiones y experiencias de investigación educativa. Barcelona: Graó.

Latorre, A. (2003). La investigación-acción. Conocer y cambiar la práctica educativa. Barcelona: Graó.

Santamaría, N. (2015). Tesis doctoral: Incidencia de las cuñas motrices y psicomotrices en la construcción de otra escuela. Universidad de Valladolid. https://www.educacion.es/teseo/mostrarRef.do?ref=1230045

Torreo, J.C. y colaboradores (2007). "Modelo Integrado de Mejora de la Convivencia". Barcelona. Graó. 
Vaca, M.J. (2002). Relatos y reflexiones sobre el tratamiento pedagógico de lo corporal en la Educación Primaria. Palencia: Asociación: Cuerpo, Educación y Motricidad.

Vaca, M.J. (1992). "Apuntes de la asignatura: Educación Física en Educación Primaria" Documento sin publicar.

Vaca, M.J.; Fuente, S. y Santamaría, N. (2013). Cuñas motrices en la escuela infantil y primaria. ISBN-13:978-84-695-8489-7 